\title{
Investigating cortisol excess or deficiency: a practical approach
}

\author{
Fahmy W F Hanna professor of endocrinology and metabolism ${ }^{12}$, Basil G Issa consultant physician \\ and endocrinologist ${ }^{3}$, Brian Kevil professor of clinical biochemistry ${ }^{3}$, Anthony A Fryer professor of \\ clinical biochemistry ${ }^{14}$
}

${ }^{1}$ University Hospital of North Midlands, Stoke-on-Trent, UK; ${ }^{2}$ Staffordshire University, Stoke-on-Trent, UK; ${ }^{3}$ Manchester University Foundation Trust, Manchester, UK; ${ }^{4}$ Institute for Applied Clinical Sciences, Keele University, Stoke-on-Trent, UK

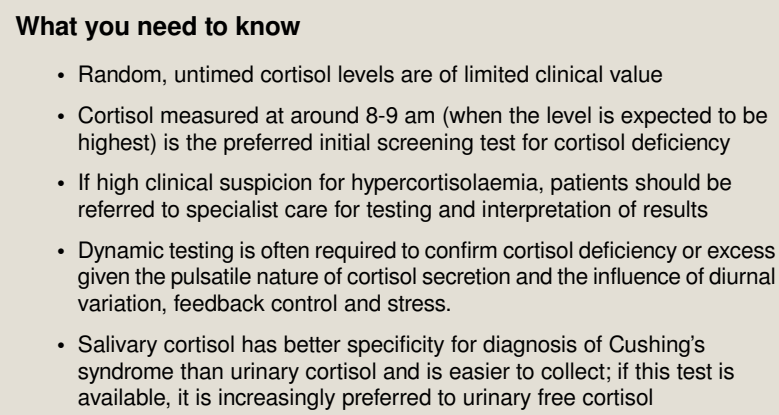

- Random, untimed cortisol levels are of limited clinical value

- Cortisol measured at around 8-9 am (when the level is expected to be highest) is the preferred initial screening test for cortisol deficiency

- If high clinical suspicion for hypercortisolaemia, patients should be referred to specialist care for testing and interpretation of results

- Dynamic testing is often required to confirm cortisol deficiency or excess given the pulsatile nature of cortisol secretion and the influence of diurnal variation, feedback control and stress.

- Salivary cortisol has better specificity for diagnosis of Cushing's syndrome than urinary cortisol and is easier to collect; if this test is available, it is increasingly preferred to urinary free cortisol

A 67 year old woman with no significant medical history required urgent orthopaedic surgery after a fall. In preparation, her bloods were collected and a random cortisol was requested, which came back at $763 \mathrm{nmol} / \mathrm{L}$. She did not have diabetes or hypertension. The anaesthetist reviewed and commented: "High cortisol, not safe for surgery until further evaluation."

A 24 year old man with previously well controlled type 1 diabetes presented with repeated hypoglycaemic episodes. Serum cortisol in clinic at 9 am came back as $143 \mathrm{nmol} / \mathrm{L}$.

Cortisol, secreted by the adrenal cortex, regulates blood pressure, glucose metabolism, and physiological responses to stress. Both cortisol over-secretion (hypercortisolism, Cushing's syndrome) and under-secretion (hypocortisolism such as in Addison's disease) are uncommon: the prevalence of Addison's disease is 6-9 per $100000,{ }^{1}$ while that of Cushing's syndrome is $4 / 100$ $000 .^{2}$ However, given the potential for life threatening consequences (such as in acute adrenal crisis) and the range of associated non-specific symptoms, it is critical for clinicians to understand how to interpret and manage cortisol status. Cortisol excess could be due to an adrenal cortisol-secreting tumour or to a functional pituitary tumour (Cushing's disease), or, less commonly, malignant tumours can secrete adrenocorticotrophic hormone (ACTH) ectopically. Cortisol deficiency could be due to primary adrenal malfunction (such as autoimmune-mediated atrophy "Addison's disease," malignant infiltration, or haemorrhage) or secondary to either pituitary or hypothalamic disease resulting in reduced secretion of ACTH.

Interpreting cortisol results can be confusing because cortisol secretion is pulsatile, shows diurnal variation, is regulated via a feedback system, and is influenced by a range of stressors. Furthermore, the tests involve measuring cortisol on its own (static testing) or as part of a stimulation or suppression test (dynamic function testing), and in a range of sample types (such as from serum, saliva, or urine).

This article is aimed at non-specialist doctors who may need to order or interpret cortisol blood tests. Grounded in cortisol physiology, it describes how you might investigate suspected cortisol deficiency and excess, the practicalities of sample choice, and correct timing to obtain meaningful results. 


\section{Sources and selection criteria}

This article is based on targeted search of online publication databases (https: //www.nlm.nih.gov/) examining the topic areas listed in the article, including:

- Cortisol physiology, including factors affecting cortisol production

- Cortisol measurement

- Biochemical identification of cortisol excess and deficiency

- Clinical presentation of cortisol excess and deficiency.

Secondary references were identified originating for those identified above, in addition to those identified through personal and professional networks (including grey literature, such as professional guidelines), and throughout the review process.

\section{Factors affecting cortisol production and measurement}

Cortisol is secreted from the adrenal cortex and is influenced by (fig 1):

Circadian rhythm-Cortisol is secreted in response to pulses of corticotrophin releasing hormone $(\mathrm{CRH})$ and $\mathrm{ACTH}{ }^{3}$ These pulses contribute to a circadian rhythm with peak cortisol levels just before waking and the nadir around bedtime. Hence, if cortisol deficiency is suspected, cortisol should be measured at around 8-9 am (when it is expected to be highest). If this is low, it would suggest deficiency. If excess cortisol is suspected, cortisol levels should be checked when they are expected to be lowest (around midnight). A random, untimed cortisol measurement is of minimal clinical value and should be avoided in assessing cortisol status.

Feedback control-Exploiting the physiologic feedback mechanisms is the underlying principle behind dynamic function tests (both stimulation and suppression tests). ${ }^{45}$ CRH produced in the hypothalamus stimulates the pituitary to produce $\mathrm{ACTH}$, which stimulates the adrenal gland to produce and release cortisol. ${ }^{36}$ Rising cortisol levels suppress further $\mathrm{CRH}$ and $\mathrm{ACTH}$ production, thereby creating a negative feedback loop. In cases of cortisol insufficiency due to adrenal causes, CRH and ACTH levels are expected to be high. However, in cases of cortisol insufficiency due to a hypothalamic or pituitary cause (such as a tumour), the ACTH level would be low. The same principles apply to cortisol excess.

Stress-Cortisol is often referred to as the stress hormone. Its secretion is promptly induced in response to a range of stressors including severe trauma, sepsis, pain, anxiety, exercise, and hypoglycaemia. The extent of impact on cortisol levels will depend on the severity of the stress. If there is suspicion of cortisol deficiency (for example, unexplained hyponatraemia, hypoglycaemia, or hypotension in a severely septic patient), interpretation of cortisol levels should take into account the underlying stress.

Cortisol binding globulin-Cortisol is bound strongly to cortisol binding globulin (CBG) in serum. Serum cortisol measurement reflects the total rather than free concentration. Factors affecting CBG, such as acute illness or medicines such as hormonal contraception, could alter reported serum cortisol without having a bearing on free cortisol concentrations. In contrast, saliva and urine samples reflect the free component of cortisol and may thus be clinically more useful. For example, in a recent study, women taking the contraceptive pill were found to have total plasma cortisol and CBG levels 2.9 and 2.6 times higher, respectively, when compared with controls, whereas 24 hour urinary free cortisol and plasma free cortisol were not significantly different. ${ }^{8}$ Given the wide availability of total serum cortisol assays (which includes the CBG-bound component), the effect of CBG may need to be taken into consideration.

Exogenous glucocorticoids used for the treatment of other diseases may also suppress the hypothalamo-pituitary-adrenal axis. This includes the use of inhaled, nasal, transdermal, and intra-articular preparations.

\section{When to consider measuring cortisol in routine clinical practice?}

Given the multi-organ impact of cortisol, its measurement may be considered in a wide range of clinical scenarios in day-to-day practice. However, adrenal disorders are uncommon. Cortisol measurement is not routinely required unless there are specific features to suspect adrenal disease. For example, rapid onset of diabetes with weight gain, easy bruising, and muscle weakness could point to possible Cushing's syndrome. Paradoxically, unexplained hypoglycaemia or hypotension developing in a patient with type 1 diabetes or thyroid disease should raise suspicion for Addison's disease. Box 1 lists some of the clinical features that should prompt a clinician to consider measuring cortisol.

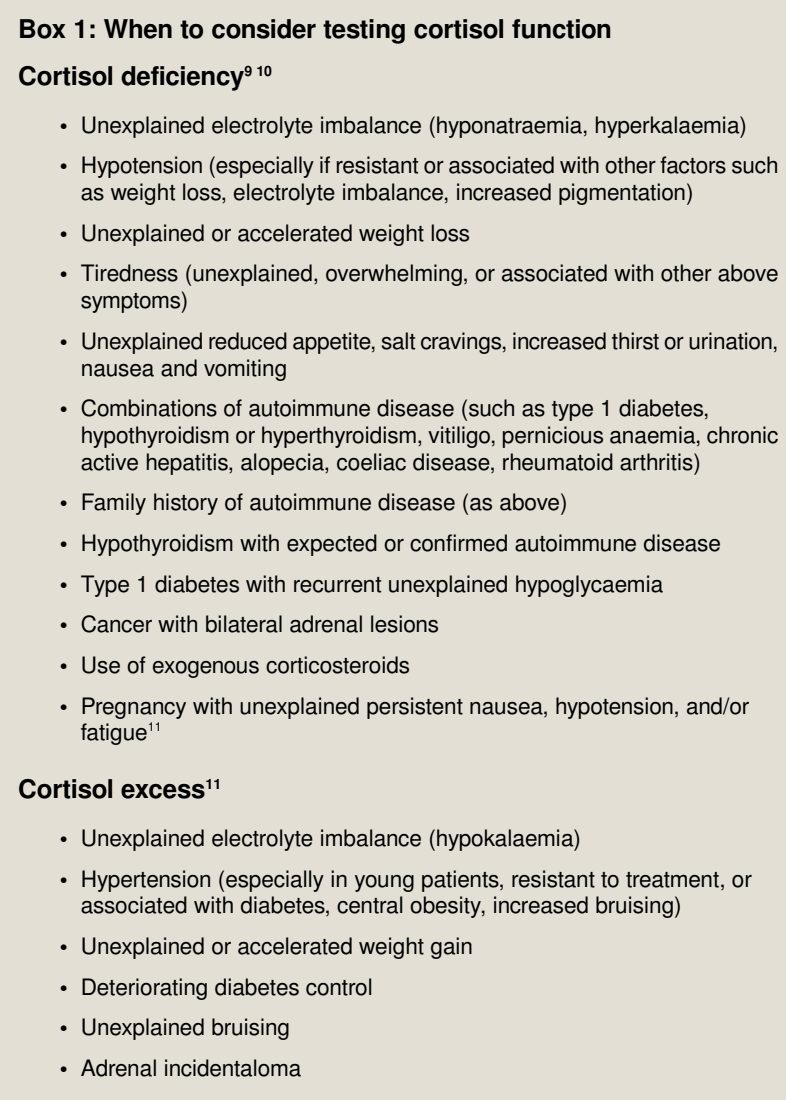

Cortisol excess could be due to a cortisol-secreting tumour or to a functional pituitary tumour (Cushing's disease) secreting ACTH that is not responsive to the normal negative feedback loop. Less often, malignant tumours can secrete ACTH ectopically. Cortisol deficiency could be due to primary adrenal malfunction (such as autoimmune-mediated atrophy "Addison's disease," malignant infiltration, or haemorrhage) or secondary to either pituitary or hypothalamic disease resulting in reduced secretion of ACTH. 


\section{What tests are available and how to interpret them}

In general, initial testing consists of static tests that are timed to correspond with physiologic diurnal cortisol variation:

- If cortisol excess is suspected, midnight cortisol should be measured

- If cortisol deficiency is suspected, cortisol levels should be checked at $8-9 \mathrm{am}$.

Cortisol testing modalities for both excess and deficiency (with their advantages and disadvantages) are summarised in table 1.

If initial static tests are abnormal, dynamic tests are then used to confirm abnormal results. The approach to testing is depicted in figure 2.

\section{Investigating suspected cortisol excess}

There are three tests that can be considered initial screening tests when investigating suspected hypercortisolism. These are midnight serum or salivary cortisol, 24 hour urinary free cortisol, and the overnight dexamethasone suppression test. Serum testing was extensively used in the past, but it requires hospitalisation and venepuncture at midnight, causing stress that could falsify the results. If available, salivary cortisol is increasingly being used for outpatient testing instead (mostly by specialists). Although an overnight dexamethasone suppression test is a dynamic test, it can be performed as an outpatient, and GPs could consider this test with select patients, particularly when clinical suspicion is low and testing can be done quickly to rule out cortisol excess.

While many of these tests can, in theory, be performed by general practitioners, if they have a high clinical suspicion of hypercortisolism, we recommend referral to specialist care for testing because of the complexity of interpreting the results and potential confounders. See table 1 for a summary of the non-dynamic tests that could be used to test for cortisol excess.

\section{Baseline (non-dynamic) tests}

Serum cortisol-A random cortisol measurement is of limited clinical value when testing for cortisol excess.

Urinary cortisol-Urinary cortisol testing relies on urine collection over 24 hours to measure the cumulative cortisol produced in that time. Although the diagnostic sensitivity and specificity vary between studies, an elevated 24 hour urinary cortisol level has been found to be $84-93 \%$ sensitive and 56-79\% specific for diagnosis of Cushing's syndrome. ${ }^{1213}$

Salivary cortisol-Salivary cortisol has better specificity than urinary cortisol and is useful for ruling out cortisol excess. A value above the reference interval has an average sensitivity of $95 \%$ and specificity of $91.6 \%$, depending on the assay used and the locally derived cut-off value, ${ }^{14}$ for the diagnosis of Cushing's syndrome. Because it is easier to collect and has the advantage of measuring free rather than bound cortisol, salivary cortisol has been advocated as a first line test. ${ }^{13}$ However, salivary cortisol assays have limited availability. On balance, clinicians have to rely on the tests that are available locally: if urinary cortisol is the only available test, then it will be the first choice, to be followed by other investigations or referrals as required.
Dynamic testing: the dexamethasone suppression test

There are different methods for performing this test. ${ }^{15}$ The overnight $1 \mathrm{mg}$ dexamethasone suppression test can be performed on an outpatient basis and consists of a patient taking $1 \mathrm{mg}$ of dexamethasone orally between 11 and $12 \mathrm{pm}$ and then a serum cortisol sample being taken at 9 am the following morning. ${ }^{16}$ A cortisol level of $<50 \mathrm{nmol} / \mathrm{L}$ has high sensitivity (95\%) and moderate specificity (80\%) for excluding Cushing's syndrome. It is important to note that some drugs can interfere with the test results by accelerating dexamethasone metabolism (such as antiepileptics and rifampicin) or impairing it (such as diltiazem and fluoxetine). Other drugs, such as oestrogens and carbamazepine, increase the concentration of cortisol binding globulin, resulting in falsely elevated cortisol levels. Other forms of the dexamethasone suppression test (such as the two day, low dose test) are available and could be considered by endocrinologists.

\section{Further testing}

Once cortisol excess is confirmed, the source of excess production along the hypothalamo-pituitary-adrenal axis (or ectopic ACTH secretion) needs to be identified, typically by an endocrinologist.

\section{Investigating suspected cortisol deficiency}

For initial screening of suspected cortisol deficiency, an 8-9 am cortisol sample is taken to measure cortisol at its expected peak. Unless the results are reassuring, further discussion or referral to endocrinology is required. See figure 2 for details.

\section{Non-dynamic serum cortisol testing}

Deficiency is highly unlikely if the 8-9 am cortisol level is above $400-475 \mathrm{nmol} / \mathrm{L}$ (the exact cut-off will vary depending on the assay used in the local laboratory). Recent work proposed that values between 336 and $506 \mathrm{nmol} / \mathrm{L}$ had a $100 \%$ specificity of predicting passing a short synacthen test. ${ }^{17}$ Cortisol levels $<150$ $\mathrm{nmol} / \mathrm{L}$ at 8-9 am warrant immediate discussion with, or referral to, endocrinology, and hydrocortisone replacement should be started urgently. Cortisol levels between 150 and $400 \mathrm{nmol} / \mathrm{L}$ (or agreed limit from the local laboratory) require further evaluation to rule out cortisol deficiency. If the sample was not collected at 8-9 am, then a repeat cortisol measurement can be performed. If the sample was collected correctly, patients with levels below 400-475 $\mathrm{nmol} / \mathrm{L}$ (depending on the local assay limit), will need follow-up testing with a specialist.

Neither urinary nor salivary cortisol has a role in the assessment of cortisol deficiency. ${ }^{18}$

\section{Dynamic tests: short synacthen test}

Synacthen mimics the effect of ACTH in stimulating cortisol production: administration of synacthen should therefore result in a predictable rise in serum cortisol. ${ }^{5}$ The short synacthen test has equivalent performance to that of the gold standard insulin stress test. ${ }^{41920}$ As it can be performed in an outpatient setting, it has become the most common dynamic test of cortisol deficiency. The cut-off values for a satisfactory response depend on the cortisol assay used ${ }^{9}: 450 \mathrm{nmol} / \mathrm{L}$ is the threshold used in many centres, though this again will need to align with the local assay. $^{21}$

Falsely reassuring test results could be obtained in a case of $(a)$ early pituitary failure before adrenal atrophy develops or $(b)$ postoperative testing in patients who had recently undergone 
surgical removal of the pituitary gland for Cushing's syndrome (as persistent adrenal hypertrophy may lead to adequate response).

\section{The role of $\mathrm{ACTH}$ measurement}

ACTH is almost always measured by specialists to determine the source of cortisol excess or deficiency along the hypothalamic-pituitary-adrenal axis. For example, in a case of cortisol excess, low ACTH levels point to an adrenal source. In practice, for the assessment of cortisol deficiency, baseline ACTH is measured only if the short synacthen test result is abnormal.

\section{Exogenous steroids and impact on cortisol measurement: a pragmatic approach}

Patients taking oral glucocorticoids at a dose higher than the physiological cortisol level (prednisolone $5 \mathrm{mg}$ or dexamethasone 1-2 mg daily) will have a suppressed pituitary-adrenal axis. They may need tapered withdrawal of their glucocorticoids (box 2), but do not need to have their cortisol concentrations measured routinely.

\section{Box 2: Tapering exogenous glucocorticoids: impact on cortisol} measurement

- Most steroid courses are short ( $<3$ weeks), and so adrenal atrophy is unlikely and cortisol measurement is not routinely required

- For longer steroid courses ( $>3$ weeks) at higher than physiological doses, gradual reduction is necessary; again cortisol testing is not required. If patients feel unwell during steroid withdrawal, they need to seek medical advice to consider more gradual withdrawal

- In cases of prolonged steroid courses (such as for polymyalgia rheumatic, which may require courses of several months), more gradual withdrawal and careful monitoring of symptoms is warranted and is sufficient in most cases. Cortisol measurement, if required, should be part of specialist evaluation

\section{Case resolution}

In the first case, given the absence of clinical features of Cushing's syndrome in this 67 year old woman with physiological stress due to injury, an elevated random cortisol level requires no further action and should not delay orthopaedic management. However, for further reassurance, we would recommend that the woman is reassessed when she is well. In the second case, the 9 am cortisol test result $<150 \mathrm{nmol} / \mathrm{L}$ required immediate further investigation to rule out cortisol insufficiency. A dynamic, confirmatory test was performed along with ACTH testing. Serum cortisol was low and ACTH was elevated, confirming the diagnosis of primary adrenal insufficiency. Starting hydrocortisone resulted in resolution of the hypoglycaemic episodes.

\section{Education into practice}

- How do you ensure accurate timing for collection of a 9 am serum cortisol sample?

- Audit the cortisol requests made in the past six months in your practice and review the timings and results in relation to the recommendations in this article.

- Reflect on the last time you considered an adrenal disorder in your practice. Would the recommendations in this article alter your management plan?
How patients were involved in the creation of this article

No patient involvement. The cases provided are fictitious but based on commonly encountered clinical scenarios.

We thank Dr Jennifer Rasanathan and Dr Kate Adlington for their insightful input and support during the review process.

Contributors: All authors substantially contributed to the conception and interpretation of the work, drafted the work, and revised it critically for important intellectual content, approved the final version of the manuscript, and agree to be accountable for all aspects of the work in ensuring that questions related to the accuracy or integrity of any part of the work are appropriately investigated and resolved. FWFH acts as guarantor.

Competing interests: We have read and understood the BMJ policy on declaration of interests and have no relevant interests to declare.

Patient consent not required (patient anonymised, dead, or hypothetical).

Provenance and peer review: Commissioned, based on an idea from the author; externally peer reviewed.

Meyer G, Neumann K, Badenhoop K, Linder R. Increasing prevalence of Addison's disease in German females: health insurance data 2008-2012. Eur J Endocrinol 2014:170:367-73. 10.1530/EJE-13-0756 24322183

2 Castinetti F, Morange I, Conte-Devolx B, Brue T. Cushing's disease. Orphanet J Rare Dis 2012;7:41. 10.1186/1750-1172-7-41 22710101

3 Weitzman ED, Fukushima D, Nogeire C, Roffwarg H, Gallagher TF, Hellman L. Twenty-four hour pattern of the episodic secretion of cortisol in normal subjects. J Clin Endocrinol Metab 1971:33:14-22. 10.1210/jcem-33-1-14 4326799

4 Hurel SJ, Thompson CJ, Watson MJ, Harris MM, Baylis PH, Kendall-Taylor P. The short Synacthen and insulin stress tests in the assessment of the hypothalamic-pituitary-adrenal axis. Clin Endocrinol (Oxf) 1996;44:141-6. 10.1046/.j.1365-2265.1996.555381.x 8849566

5 Nugent CA, Nichols T, Tyler FH. Diagnosis of Cushing's syndrome-single dose dexamethasone suppression test. Arch Intern Med 1965;116:172-6. 10.1001/archinte.1965.03870020012006 14315650

6 Hanna FWF, Issa BG, Sim J, Keevil B, Fryer AA. Management of incidental adrenal tumours. BMJ 2018;360:j5674. 10.1136/bmj.j5674 29348269

7 Russell G, Lightman S. The human stress response. Nat Rev Endocrinol 2019;15:525-34. 10.1038/s41574-019-0228-0 31249398

8 Jung C, Ho JT, Torpy DJ, etal . A longitudinal study of plasma and urinary cortisol in pregnancy and postpartum. J Clin Endocrinol Metab 2011;96:1533-40.

10.1210/jc.2010-2395 21367926

9 Bornstein SR, Allolio B, Arlt W, etal . Diagnosis and treatment of primary adrenal insufficiency: an Endocrine Society Clinical Practice Guideline. J Clin Endocrinol Metab 2016;101:364-89. 10.1210/jc.2015-1710 26760044

10 Pazderska A, Pearce SH. Adrenal insufficiency-recognition and management. Clin Med (Lond) 2017;17:258-62. 10.7861/clinmedicine.17-3-258 28572228

11 Nieman LK, Biller BMK, Findling JW, etal . The diagnosis of Cushing's syndrome: an Endocrine Society Clinical Practice Guideline. J Clin Endocrinol Metab 2008;93:1526-40. 10.1210/jc.2008-0125 18334580

12 Aranda G, Careaga M, Hanzu FA, etal . Accuracy of immunoassay and mass spectrometry urinary free cortisol in the diagnosis of Cushing's syndrome. Pituitary 2016;19:496-502. 10.1007/s11102-016-0730-5 27259502

13 Elias PCL, Martinez EZ, Barone BFC, Mermejo LM, Castro M, Moreira AC. Late-night salivary cortisol has a better performance than urinary free cortisol in the diagnosis of Cushing's syndrome. J Clin Endocrinol Metab 2014;99:2045-51. 10.1210/jc.2013-4262 24628557

14 Zhang Q, Dou J, Gu W, Yang G, Lu J. Reassessing the reliability of the salivary cortisol assay for the diagnosis of Cushing syndrome. J Int Med Res 2013;41:1387-94. $10.1177 / 030006051349801724065452$

15 Liddle GW. Tests of pituitary-adrenal suppressibility in the diagnosis of Cushing's syndrome. J Clin Endocrinol Metab 1960;20:1539-60. 10.1210/jcem-20-12-1539 13761950

16 Wood PJ, Barth JH, Freedman DB, Perry L, Sheridan B. Evidence for the low dose dexamethasone suppression test to screen for Cushing's syndrome-recommendations for a protocol for biochemistry laboratories. Ann Clin Biochem 1997;34:222-9. 10.1177/000456329703400302 9158818

17 Sbardella E, Isidori AM, Woods CP, etal . Baseline morning cortisol level as a predictor of pituitary-adrenal reserve: a comparison across three assays. Clin Endocrinol (Oxf) 2017;86:177-84. 10.1111/cen.13232 27616279

18 Elder CJ, Harrison RF, Cross AS, etal . Use of salivary cortisol and cortisone in the highand low-dose synacthen test. Clin Endocrinol (Oxf) 2018;88:772-8. 10.1111/cen.13509 29106701

19 Lindholm J, Kehlet $\mathrm{H}$. Re-evaluation of the clinical value of the $30 \mathrm{~min}$ ACTH test in assessing the hypothalamic-pituitary-adrenocortical function. Clin Endocrinol (Oxf) 1987;26:53-9. 10.1111/j.1365-2265.1987.tb03638.x 3026692

20 Stewart PM, Corrie J, Seckl JR, Edwards CR, Padfield PL. A rational approach for assessing the hypothalamo-pituitary-adrenal axis. Lancet 1988;1:1208-10. 10.1016/S0140-6736(88)92020-X 2897016

21 Ueland GÅ, Methlie P, Øksnes M, etal . The short cosyntropin test revisited: new normal reference range using LC-MS/MS. J Clin Endocrinol Metab 2018;103:1696-703. 10.1210/jc.2017-02602 29452421

Published by the BMJ Publishing Group Limited. For permission to use (where not already granted under a licence) please go to http://group.bmj.com/group/rights-licensing/ permissions 
Table

Table 1| Advantages and disadvantages of different options for cortisol sampling

\begin{tabular}{|c|c|c|c|}
\hline Procedure & Requirements & Advantages & Disadvantages \\
\hline \multicolumn{4}{|l|}{ Serum sample } \\
\hline $\begin{array}{l}\text { - Random, untimed } \\
\text { cortisol is of limited } \\
\text { clinical value }\end{array}$ & $\begin{array}{l}\text { - Early morning (8-9 am) } \\
\text { sample for assessing cortisol } \\
\text { deficiency } \\
\text { - Late evening ( } 11-12 \mathrm{pm}) \\
\text { sample for assessing cortisol } \\
\text { excess }\end{array}$ & $\begin{array}{l}\text { - Test widely available } \\
\text { - Well characterised cut-off } \\
\text { concentrations }\end{array}$ & $\begin{array}{l}\text { - Invasive test (venepuncture)-Could lead to stress induced } \\
\text { hypercortisolaemia, especially if sample collected at midnight } \\
\text { - Measures total, not free cortisol, so vulnerable to abnormalities } \\
\text { in cortisol binding globulin (CBG): } \\
\text { - During critical illness, CBG may fall and the consequent low total } \\
\text { cortisol may be mistaken for adrenal insufficiency } \\
\text { - Oral contraceptives (oestrogen component) or pregnancy result } \\
\text { in increased CBG, spuriously raising total cortisol levels (but not } \\
\text { influencing free cortisol) } \\
\text { - Interference from exogenous steroids in some assays } \\
\text { - Interference from cortisol precursors in some assays }\end{array}$ \\
\hline \multicolumn{4}{|l|}{ Saliva sample } \\
\hline $\begin{array}{l}\text { - Better specificity for } \\
\text { diagnosis of Cushing's } \\
\text { syndrome than urinary } \\
\text { cortisol and easier to } \\
\text { collect }\end{array}$ & $\begin{array}{l}\text { - Use synthetic swab or } \\
\text { passive drool }(0.5 \mathrm{~mL})\end{array}$ & $\begin{array}{l}\text { - Non-invasive test } \\
\text { - Home collection possible } \\
\text { - Convenient for midnight samples } \\
\text { - Measures free cortisol } \\
\text { - Overcomes stress induced } \\
\text { hypercortisolaemia } \\
\text { - Stability at } 4^{\circ} \mathrm{C} \text { for } 2 \text { weeks } \\
\text { - Food and blood contamination have } \\
\text { no impact if sampling instructions are } \\
\text { followed }\end{array}$ & $\begin{array}{l}\text { - Patients taking diuretics may find it difficult to collect enough } \\
\text { saliva (dry mouth) } \\
\text { - Food or blood contamination (collect } \geq 60 \text { min after eating or } \\
\text { cleaning teeth) } \\
\text { - Restricted availability-May need to send samples to specialist } \\
\text { laboratories, with implications for cost and turnaround times } \\
\text { - Only of use in identifying cortisol excess } \\
\text { - Potential for "user error" in self sampling of saliva }\end{array}$ \\
\hline \multicolumn{4}{|l|}{ Urine sample } \\
\hline & - 24 hour collection & $\begin{array}{l}\text { - Non-invasive test } \\
\text { - Outpatient test } \\
\text { - Measures free cortisol } \\
\text { - Measurement widely available }\end{array}$ & $\begin{array}{l}\text { - Samples may not represent a full } 24 \text { hour collection } \\
\text { - Metabolites may interfere with some assays } \\
\text { - Only of use in identifying cortisol excess } \\
\text { - Lower sensitivity and specificity for ruling out hypercortisolaemia } \\
\text { than other methods }\end{array}$ \\
\hline
\end{tabular}




\section{Figures}

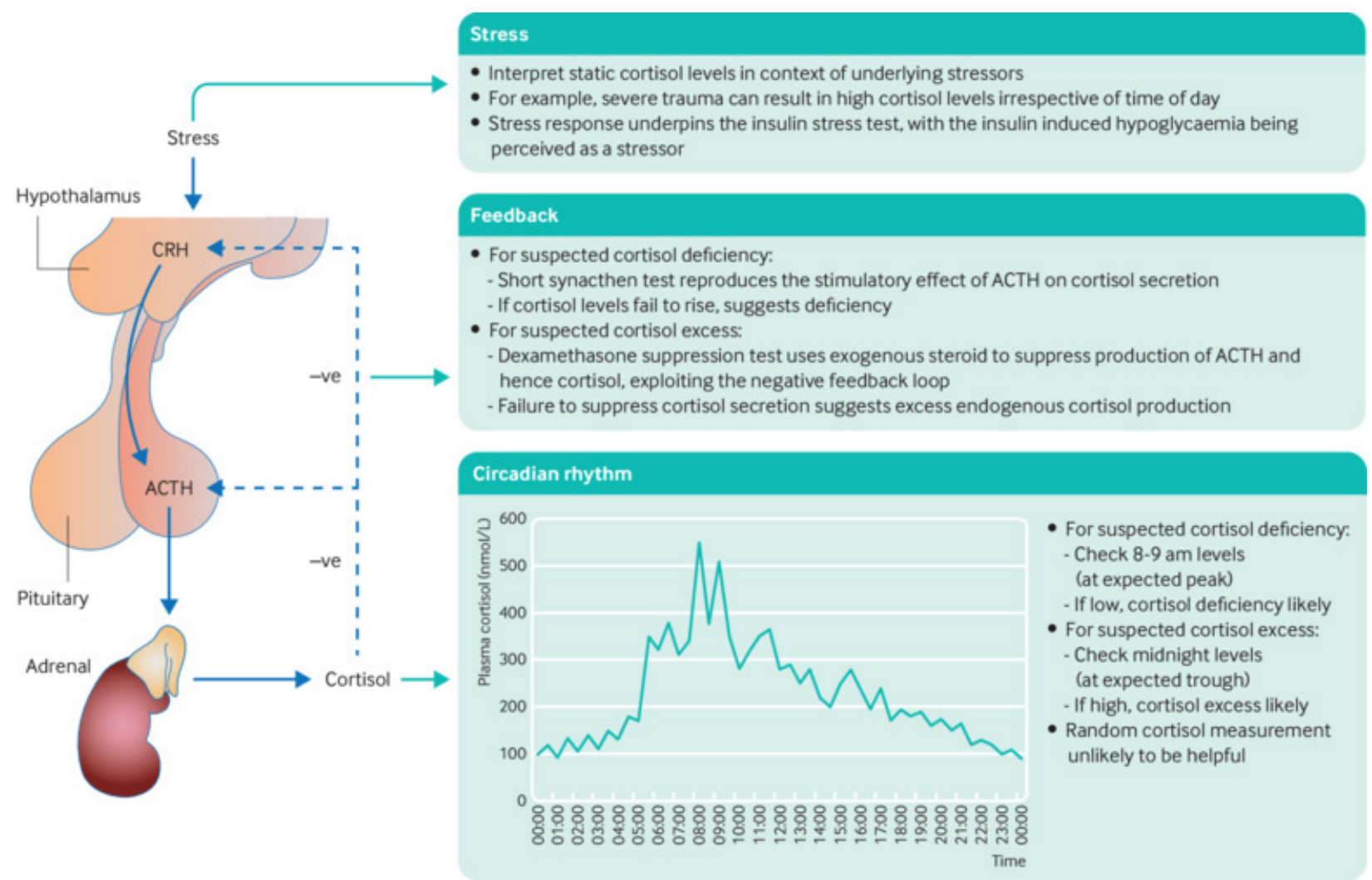

Fig 1 How cortisol testing is affected by its underlying physiology 


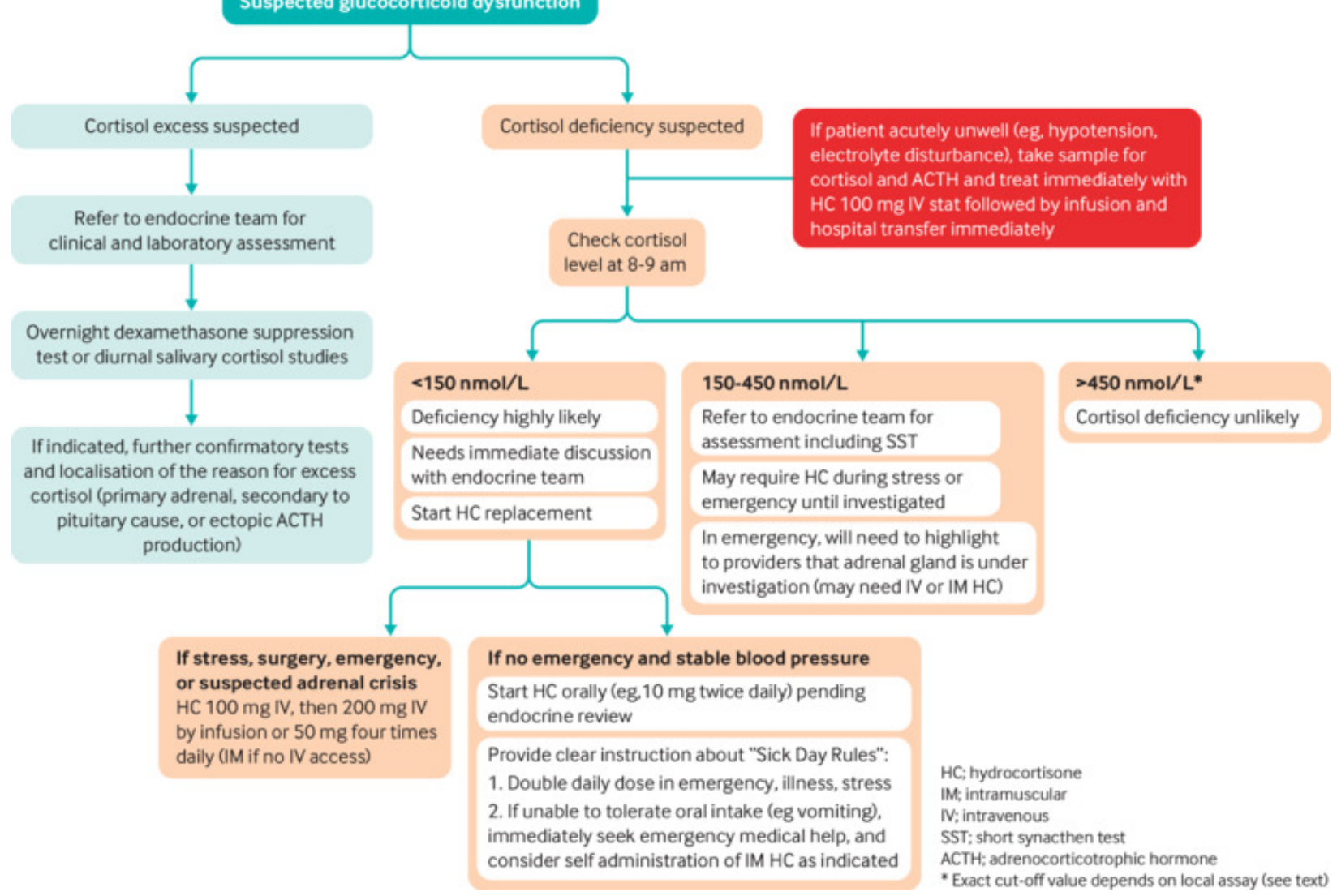

Fig 2 Action plan for suspected glucocorticoid dysfunction 